International Journal of Pure and Applied Mathematics

Volume 97 No. 4 2014, 395-407

ISSN: 1311-8080 (printed version); ISSN: 1314-3395 (on-line version)

url: http://www.ijpam.eu

doi: http://dx.doi.org/10.12732/ijpam.v97i4.2

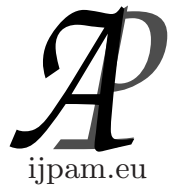

\title{
EVEN SEQUENTIAL HARMONIOUS LABELING OF SOME CYCLE RELATED GRAPHS
}

\author{
P. Selvaraju ${ }^{1}$, P. Balaganesan ${ }^{2}$, L. Vasu ${ }^{3}$, M.L. Suresh ${ }^{4}$ \\ ${ }^{1}$ Department of Mathematics \\ Vel Tech Multi Tech Dr. Rangarajan Dr. Sankanthula \\ Engineering College \\ Chennai, 600 062, INDIA \\ ${ }^{2,4}$ Hindustan University \\ Chennai, 603 103, INDIA \\ ${ }^{3}$ Department of Mathematics \\ Easwari Engineering College \\ Chennai, 600 089, INDIA
}

\begin{abstract}
In this paper, we generalize this result on cycles by showing that the $k C_{n}$ snake with string $1,1, \cdots, 1$ when $n \equiv 0(\bmod 4)$ are even sequential harmonious graph. Also we show that the $k C_{4}$ snake with $m$-pendant edges for each $k, m \geq 1$, (for linear case and for general case). Moreover, we show that, all subdivision of $2 m_{k}$ - snake are even sequential harmonious for each $k, m \geq 1$ . Finally we present some examples to illustrate the proposed theories.
\end{abstract}

AMS Subject Classification: $05 \mathrm{C} 78$

Key Words: even sequential harmonious labeling, pendant edges, cyclic snakes, subdivision double triangular snakes

\section{Introduction}

Graph labeling is an active area of research in graph theory which has mainly

Received: December 19, 2013

(C) 2014 Academic Publications, Ltd.

$\S$ Correspondence author url: www.acadpubl.eu 
evolved through its many applications in coding theory, communication networks, mobile telecommunication system. Optimal circuits layouts or graph decompositions problems, no name just a few of them.

Most graph labeling methods trace their origin to one introduced by Rosa [1] called such a labeling a $\beta$-valuation and Golomb subsequently called graceful labeling, and one introduced by Graham and Sloane [2] called harmonious labeling. Several infinite families of graceful and harmonious graphs have been reported. Many illustrious works on graceful graphs brought a tide to different ways of labeling the elements of graph such as odd graceful.

All graphs in this paper are finite, simple graphs and undirected graph. The symbols $V(G)$ and $E(G)$ denote the vertex set and edge set of a graph $G$. The cardinality of the vertex set is called the order of $G$. The cardinality of the edge set is called the size of $G$. A graph with $p$ vertices and $q$ edges is called a $G(p, q)$ graph [4].

A graph $G$ of size $q$ is odd-graceful, if there is an injection $f: V(G) \rightarrow$ $\{0,1,2,, 2 q-1\}$ such that, when each edge $x y$ is assigned the label or weight $f(x)-f(y)$, the resulting edge labels are $\{1,3,5, \cdots, 2 q-1\}$. This definition was introduced by Gnanajothi. Many researchers have studied odd graceful labeling. Seoud and Abdel-Aal determine all connected odd graceful graphs of order less than or equal to 6 . For a dynamic survey of various graph labeling problems we refer to Gallian [3]. A graph $G$ is said to be odd harmonious if there exists an injection $f: V(G) \rightarrow\{0,1,2, \cdots, 2 q-1\}$ such that the induced function $f^{*}: E(G) \rightarrow\{1,3, \cdots, 2 q-1\}$ defined by $f^{*}(u v)=f(u)+f(v)$ is a bijection. Then $f$ is said to be an odd harmonious labeling of $G$. [8]. A graph $G$ with $p$ vertices and $q$ edges is said to have an even sequential harmonious labeling if there exists a function $f: V(G) \rightarrow\{0,1,2, \cdots, 2 q\}$ such that the induced map $f^{*}: E(G) \rightarrow\{2,4,6, \cdots, 2 q\}$ defined by

$$
\begin{aligned}
f^{*}(u v)=f(u)+f(v), & \text { if } f(u)+f(v) \text { is even } \\
f^{*}(u v)=f(u)+f(v)+1, & \text { if } f(u)+f(v) \text { is odd, }
\end{aligned}
$$

then the resulting edge labels are distinct. A graph $\mathrm{G}$ is said to be an even sequential harmonious graph if it admits an even sequential harmonious labeling.

Consider a Graph $k C_{n^{-}}$snake with $k \geq 2$. Let $u_{1}, u_{2}, \cdots, u_{k-1}$ be the consecutive cut vertices of $G$. Let $d_{i}$ be the distance between $u_{i}$ and $u_{i+1}$ in $G$,and the string $\left(d_{1}, d_{2}, \cdots, d_{k-2}\right)$ of integers. Hence, any graph $G=k C_{n}$ -snake, can be represented by a string. For instance the string (from left to right) of the $5 C_{4^{-}}$snake of figure 4 is $2,2,2,2,2$. Gracefulness of the kind of $k C_{4}$-snake studied by Gnanajothi have string $2,2, . .2$. We obtain in theorem 
2.4 an even sequential harmonious labeling of the snake $1,1 \cdots, 1$ if the string o given $k C_{n}$-snake is , $\left\lfloor\frac{n}{2}\right\rfloor, \cdots,\left\lfloor\frac{n}{2}\right\rfloor$, we say that snake $k C_{n}$ is linear.

This paper can be divided into two sections. Section 1, we show that the graphs $k C_{4}$-snake $\odot m k_{1}$ (the graph obtained by joining $m$ pendant edges to each vertex of $k C_{4}$-snake $k, m \geq 1$ ) for linear and general cases of $k C_{4}$-snake for each $k \geq 1$ are even sequential harmonious. We also obtain an even harmonious labeling of $k C_{n}$ with the sequences string is $1,1, \cdots, 1$ and when $n \equiv 0(\bmod 4)$ in section 2, we show that an even sequential harmonious labeling of the all subdivision of of double triangular snakes finally, we prove that the all Subdivision of $2 m \triangle_{k}$ snake are even sequential harmonious for each $k, m>1$.

\section{Main Results}

Theorem 2.1. The linear graphs $k C_{4^{-}}$snake $\odot m k_{1}$ are even sequential harmonious for $k, m \geq 1$.

Proof. Consider the linear graph $G=k C_{4}$-snake, $k \geq 1$ which has the vertices $w_{i}, u_{j}$ and $v_{j}$ where $i=0,1,2, \cdots, k, j=1,2,3, \cdots, k$. In order to get the linear $k C_{4}$ - snake $\odot m k_{1}, k, m \geq 1$, we add $m$ pendant edges $w_{i}^{l}, u_{j}^{l}$ and $v_{j}^{l}$ to each vertex of $w_{i}, u_{j}, v_{j}$ respectively such that $l=0,1,2, \cdots, m$. Now, let $G$ be the linear $k C_{4}$ - snake $\odot m k_{1}, k, m \geq 1$ be described as indicated in figure 1 .

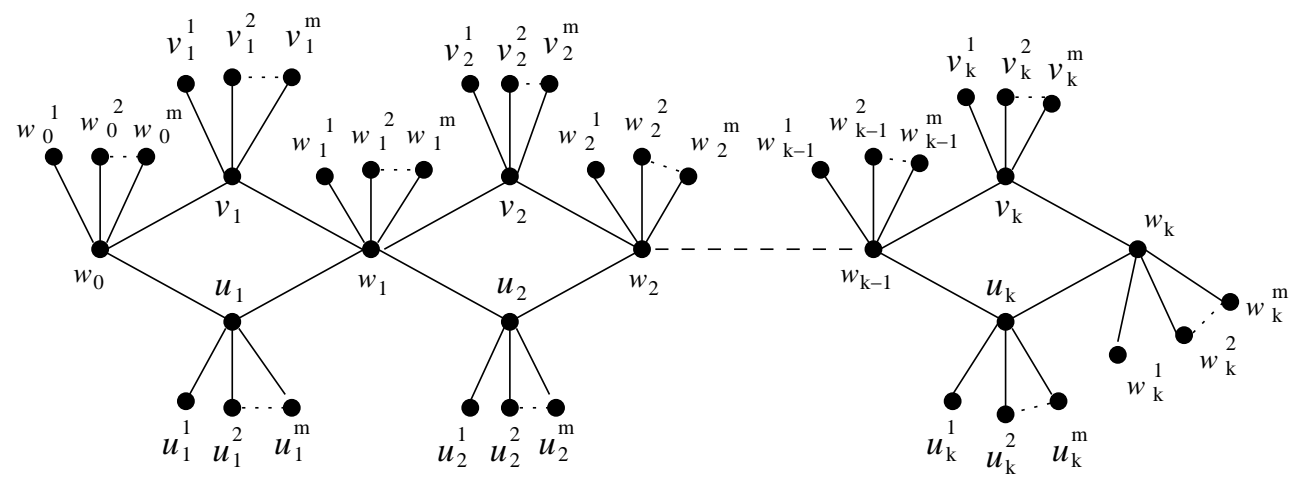

Figure 1

It is clear that the number of edges of the graph $G$ is $q=4 k+m(3 k+1)$. We define the labeling function $f: V(G) \rightarrow\{0,1,2, \cdots, 8 k+2 m(3 k+1)$ as 
follows:

$$
\begin{array}{r}
f\left(w_{i}\right)=4 i ; \quad 0 \leq i \leq k, \\
f\left(v_{i}\right)=4 i-3 ; \quad 1 \leq i \leq k, \\
f\left(u_{i}\right)=4 i-1 ; \quad 1 \leq i \leq k, \\
f\left(w_{i}^{j}\right)=2[q-j-(m+2) i]+1, \quad 0 \leq i \leq k, \quad 1 \leq j \leq m, \\
f\left(u_{i}^{j}\right)=4 k(m+2)-2 j-4(m+1) i+2 m+2,1 \leq i \leq k, \quad 1 \leq j \leq m, \\
f\left(v_{i}^{j}\right)=4 k(m+2)-2 j-4(m+1)(i-1), 1 \leq i \leq k, \quad 1 \leq j \leq m .
\end{array}
$$

Let $\mathrm{A}, \mathrm{B}, \mathrm{C}, \mathrm{D}, \mathrm{E}$ denote the edge set.

$$
\begin{aligned}
A & =\left\{e_{i}=w_{i-1} v_{i}, f\left(e_{i}\right)=8 i-6: 1 \leq i \leq k\right\}, \\
B & =\left\{e_{i}=w_{i-1} u_{i} / e_{i}=8 i-4: 1 \leq i \leq k\right\}, \\
C & =\left\{e_{i}=v_{i} w_{i} / e_{i}=8 i-2: 1 \leq i \leq k\right\}, \\
D=\left\{e_{i}{ }_{i}^{u} w_{i} / e_{i}=8 i: 1\right. & \leq i \leq k\} .
\end{aligned}
$$

The remaining odd edge labels from $8 k+l$ to $2 k+2 m(3 k+l)$ are obtained from the following:

$$
\begin{aligned}
& E=\left\{e_{j}=u_{j} u_{j}^{j} / f\left(e_{j}\right)=4 k(m+2)-2 j-4 m i+2 m+2,1 \leq i \leq k, 1 \leq j \leq m\right\}, \\
& F=\left\{e_{j}=v_{j} v_{j}^{j} / f\left(e_{j}\right)=4 k(m+2)-2 j-4 m i+4 m+2,1 \leq i \leq k, 1 \leq j \leq m\right\}, \\
& G=\left\{e_{j}=w_{i} w_{i}^{j} / f(e j)=2[q-j-m i]+2,0 \leq i \leq k, 1 \leq j \leq m\right\} .
\end{aligned}
$$

Hence the graph $G$ is an even sequential harmonious.

Example 2.2. An even sequential harmonious labeling of the graph $3 C_{4^{-}}$ snake $\odot 2 k_{1}$ is shown in figure 2 .

Theorem 2.3. The following graphs are an even sequential harmonious.

(i) $k C_{4}$ - snake for each $k \geq 1$.

(ii) $k C_{4}$ - snake $\odot m k_{1}$ for each $k, m \geq 1$.

Proof. The graph $k C_{4}$ - snake can be considered as a bipartite graph (one partite set has black vertices and the other has white vertices) it is possible to embed it, on a square grid as is showed in the figure 3.

Let $G$ be the graph $k C_{4}$ - snake $\odot m k_{1}$ which obtained by joining $m$ - pendant edges to each vertex of $k C_{4}$ - snake. Then $|V(G)|=(m+1)(3 k+1)$ and $|E(G)=4 k+m(3 k+1)|$. Now, we are running the following steps sequentially 


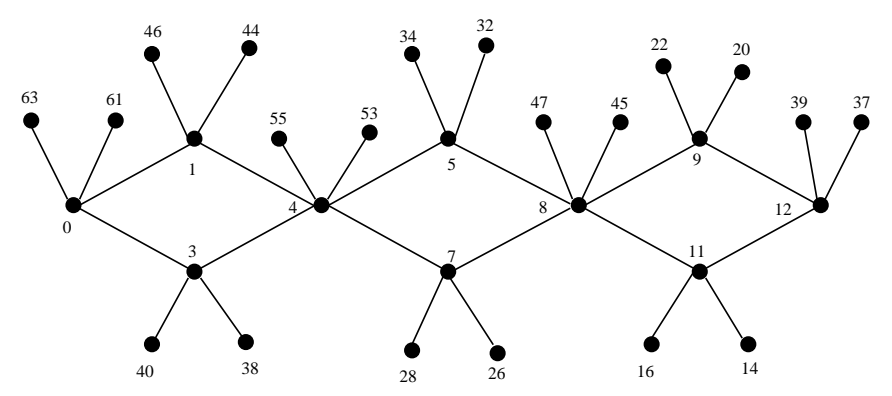

Figure 2: The graph linear $3 C_{4}$ - snake $\odot 2 k_{1}$ with its even sequential harmonious labeling

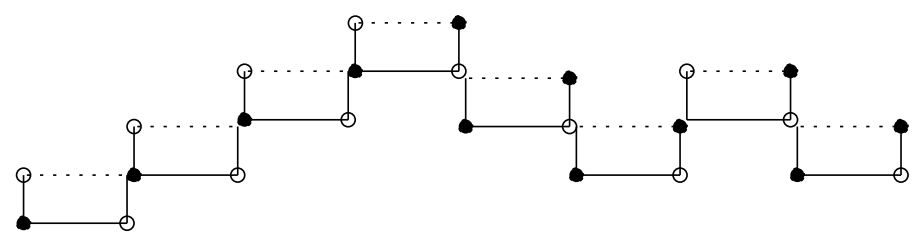

Figure 3

in order to prove the Theorem ;

Step 1. Since $k C_{4}$-snake is a bipartite graph so it has one partite set has black vertices and the other has white vertices as is showed in figure 3. Put black vertices in string ordered by diagonals from left to right and inside each diagonal from bottom to top, assign to them from an arithmetic progression of difference 4 which first term is zero, when we move to another diagonal we use an arithmetic progression of difference 2, counting until the last black vertex has been numbered. Similarly, put the White vertices on a string ordered for diagonals from left to right and inside each diagonal from top to bottom, starting with the first diagonal assign numbers from an arithmetic progression of difference 2 which first term is 1 , when we move to another diagonal we use an arithmetic progression of differences 2 counting until the last white vertex has been numbered.

Step 2. In this step, We are labeling the vertices of $m$-pendant edges which contact with the white diagonals, from the right to left and inside each white diagonal from bottom to top, assign to them from an arithmetic progression of 
difference 2, which first term is $z$ such that $z=y+2$ where $y$ is the last vertex labeling of black diagonal. when we move to a new vertex of the white diagonal, the first vertex of m-pendant edges is labeled by an arithmetic progression of difference 4 , but the arithmetic progression of difference 2 has been used with the remain $(m-l)$ vertices of $m$-pendant edges. We move from a vertex to another of the white diagonals until the last white vertex.

Step 3.Finally we are labeling the vertices of $m$-pendant edges which contact with the black diagonals, from left to right and inside each black diagonal from bottom to top, assign to them from an arithmetic progression of difference $(-2)$, which first term is $(2 q-1)$ where $q$ is the size of $G$, When to move to a new vertex of the black diagonal, the first vertex of $m$-pendant edges is labeled by an arithmetic progression of difference (-6) but the arithmetics progression of difference $(-2)$ has been used with the remain $(m-l)$ vertices of $m$-pendant edges. We move from a vertex to another of the black diagonal until the last black vertex.

Now, we have complete the proof by running the above steps, we mention only the vertices labels and the reader can fulfill the proof as we did in the previous theorem where step 1 give us an even sequential harmonious labeling of the graph $k C_{4}$ - snake for each $k \geq 1$, and step 1-step 3 give us an even sequential harmonious labeling of the graph $k C_{4}$-snake $\odot m k_{1}$ for each $k, m \geq 1$ (in general case).

The following example illustrates the last result.

Example 2.4. An even sequential harmonious labeling of the graph $5 C_{4-}$ snake $\odot 2 k_{1}$ (for general case) is shown in Figure 4 .

The graphs $k C_{4}$-snake when the sequence string is $(1,1,1, \cdots, 1)$ then $n \equiv$ $0(\bmod 4)$ are studied in the following Theorem:

Theorem 2.5. The graphs $k C_{4 m}$-snake for each $k, m \geq 1$, with string $(1,1, \cdots, 1)$ are even sequential harmonious.

Proof. Let $G=k C_{4 m}$-snake $=(n-1) C_{4 m}$-snake can be described as indicated in Figure 5.

It is clear that $|E(G)|=4 m(n-1)$. We define the labeling function $f: V(G) \rightarrow\{0,1,2, \cdots, 8 m(n-1)$ as follows:

$f\left(v_{i}^{1}\right)=\left\{\begin{array}{l}4 m(i-1), i=1,3,5, \cdots, n \text { or } n-1 \\ 4 m(i-1)+1, i=2,4,6, \cdots, n-1 \text { or } n .\end{array}\right.$ 


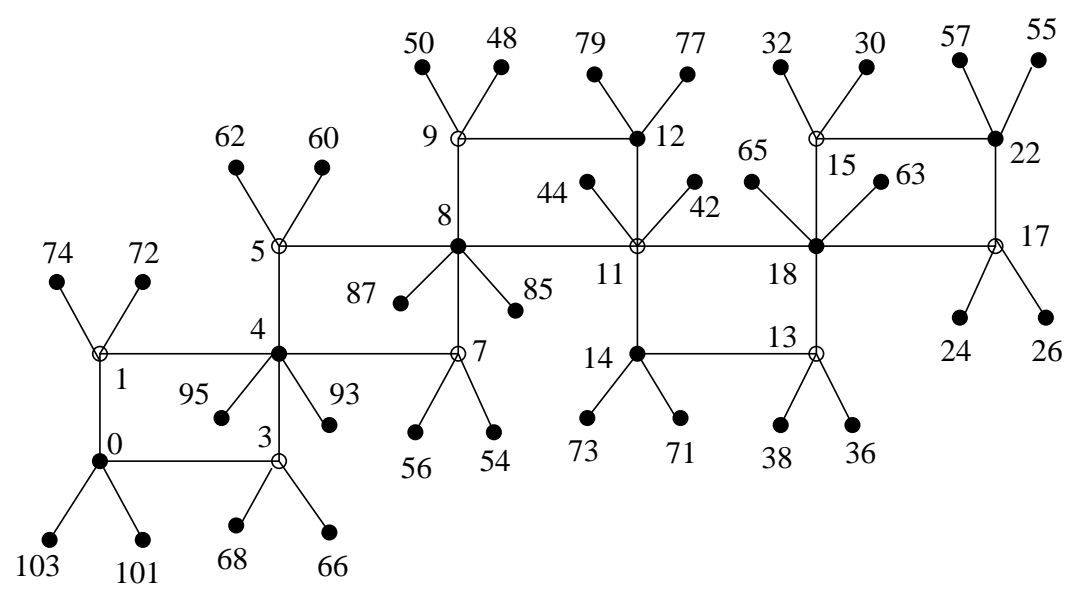

Figure 4: The graph $5 C_{4}$ - snake $\odot 2 k_{1}$ (for general case) with its even sequential harmonious labeling

$f\left(v_{1}^{j}\right)=j-1,1 \leq j \leq 2 m$.

For labeling the vertices $v_{i 1}^{j}, 2 \leq i \leq n, 2 \leq j \leq 2 m$.

We consider the following two cases:

Case i: If $i$ is odd, $3 \leq i \leq n$, we have the following labeling,for each $2 \leq j \leq 2 m$ $f\left(v_{i 1}^{j}\right)=f\left(v_{i}^{1}\right)-j+1$.

Case ii: If $i$ is even, $2 \leq i \leq n$, we have the following labeling,for each $2 \leq j \leq 2 m$ :

$f\left(v_{i 1}^{j}\right)=\left\{\begin{array}{l}f\left(v_{i}^{1}\right)-j+1, i=1,3,5, \cdots, 2 m-1 \\ f\left(v_{i}^{1}\right)-j-1, i=2,4,6, \cdots, 2 m\end{array}\right.$.

Now we label the remaining vertices $v_{i 2}^{j}, 2 \leq i \leq n-1,1 \leq j \leq 2 m$ as follows:

$f\left(v_{i 2}^{j}\right)=f\left(v_{i 1}^{1}+2(j-1), 1 \leq j \leq 2 m, 2 \leq i \leq n-1\right.$.

It follows that $f$ admits an even sequential harmonious labeling for $(n-1) C_{4} m$ - snake. Hence $(n-1) C_{4} m$ - snake for each $n \geq 2, m \geq 1$ with the string $(1,1, \cdots, 1)$ are even sequential harmonious graphs.

Example 2.6. Even sequential harmonious labeling of graph $4 C_{8}$ - snake with the string $(1,1, \cdots, 1)$ is shown in Figure 6 . 


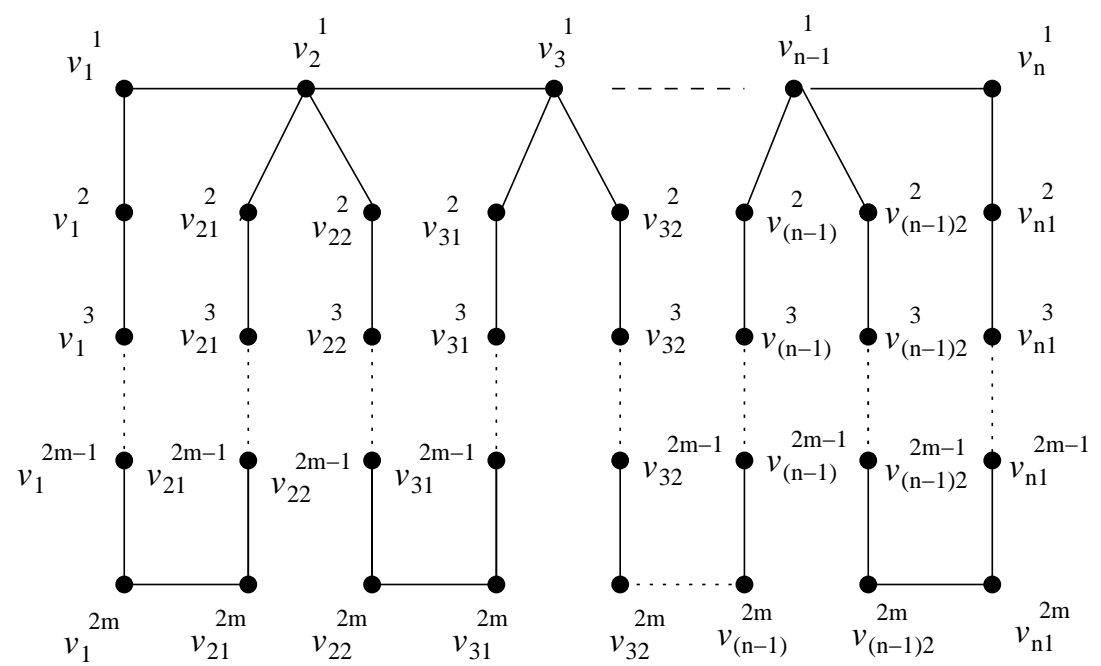

Figure 5

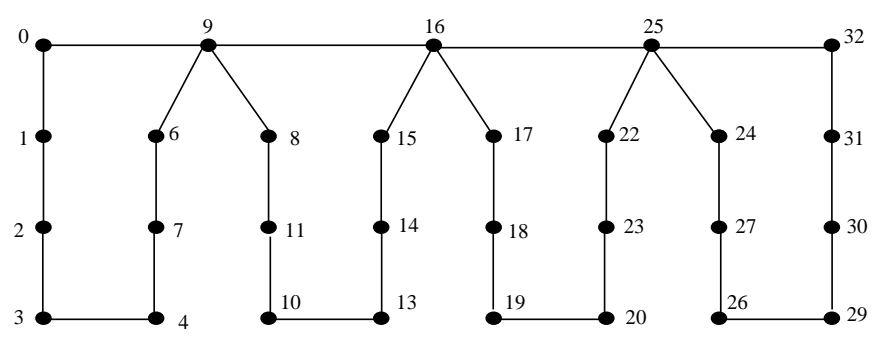

Figure 6: The graph $4 C_{8}$ - snake with its even sequential harmonious labeling

\section{Subdivision of Double Triangles Snake}

Rosa [8] defined a triangular snake (or $\triangle$ - snake) as a connected graph in which all blocks are triangles and the block cut point graph is a path. Let $\triangle_{k}$ - snake be a $\triangle$-snake with $k$ blocks while $n \triangle_{k}$ - snake be a $\triangle$-snake with $k$ blocks and every block has $n$ number of triangles with one common edge.

David moulton [9] proved that triangular snakes with $p$ triangles are graceful if $p$ is congruent to 0 or 1 modulo 4 . $\mathrm{Xu}[10]$ proved that they are harmonious if and only if $p$ is congruent to 0,1 or 3 modulo 4 . A double triangular snake is a graph that formed by two triangular snakes have a common path. The 
harmonious labeling of double triangle snake introduced by Xi Yue et al [6]. It is known that, the graphs which contain odd cycles are not even sequential harmonious so we used the subdivision notation for odd cycle in order to prove that all subdivision of double triangular snakes are even sequential harmonious.

Theorem 3.1. All subdivision of double triangular snakes $\left(2 \triangle_{k}\right.$ - snake, $k \geq 1$ ) are even sequential harmonious.

Proof. Let $G=2 \triangle_{k}$-snake has $q$ edges and $p$ vertices. The graph $G$ consists of the vertices $\left(u_{1}, u_{2}, \cdots, u_{k+1}\right),\left(v_{1}, v_{2}, \cdots, v_{k}\right)$, $\left(w_{1}, w_{2}, \cdots, w_{k}\right)$. Therefore we get the subdivision of double triangular snakes $S(G)$ by subdividing every edge of double triangular $2 \triangle_{k}$-snake exactly once. Let $y_{i}$ be the newly added vertex between $u_{i}$ and $u_{i+1}$ while $w_{i 1}$ and $w_{i 2}$ are newly added vertices between $w_{i} u_{i}$ and $w_{i} u_{i+1}$ respectively where $1 \leq i \leq k$. Finally $v_{i 1}$ and $v_{i 2}$ are newly added vertices between $v_{i} u_{i}$ and $v_{i} u_{i+1}$ respectively, such that $1 \leq i \leq k$. Let the graph $S(G)$ as described as indicated in Figure 7.
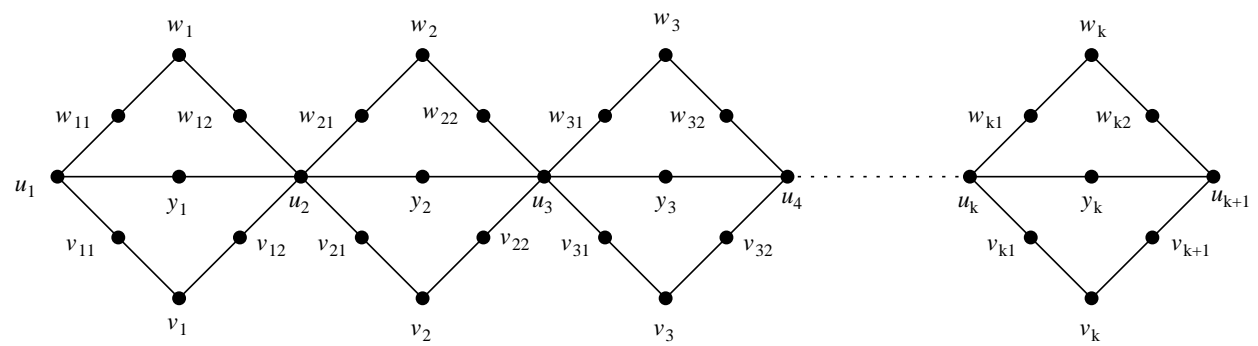

Figure 7: The subdivision of double triangular snakes $\left(2 \triangle_{k}\right.$-snake)

It is clear that the number of edges of the graph $S(G)$ is $10 k$. We define the labeling function : $f: V(S(G)) \rightarrow\{0,1,2, \cdots, 20 k\}$ as follows:

$f\left(u_{i}\right)=6(i-1), 1 \leq i \leq k+1=n$,

$f\left(y_{i}\right)=14 i-11,1 \leq i \leq k=n-1$,

$f\left(w_{i}\right)=6 i+4,1 \leq i \leq k=n-1$,

$f\left(v_{i}\right)=6 i-4,1 \leq i \leq k=n-1$,

$f\left(w_{i j}\right)=14 i+8 j-21,1 \leq i \leq k=n-1, j=1,2$,

$f\left(v_{i j}\right)=14 i+6 j-15,1 \leq i \leq k=n-1, j=1,2$.

The edge labels will be as follows:

- The vertices $u_{i}$ and $v_{i 1}, 1 \leq i \leq k$, induce the edge labels $\{20 i-18,1 \leq i \leq k\}=\{2,22, \cdots, 20 k-18\}$.

- The vertices $u_{i}$ and $y_{i}, 1 \leq i \leq k$, induce the edge labels 
$\{20 i-16,1 \leq i \leq k\}=\{4,24, \cdots, 20 k-16\}$.

- The vertices $u_{i}$ and $v_{i 1}, 1 \leq i \leq k$, induce the edge labels $\{20 i-14,1 \leq i \leq k\}=\{6,26, \cdots, 20 k-14\}$.

- The vertices $v_{i 1}$ and $v_{i}, 1 \leq i \leq k$, induce the edge labels $\{20 i-12,1 \leq i \leq k\}=\{8,28, \cdots, 20 k-12\}$.

- The vertices $y_{i}$ and $u_{i+1}, 1 \leq i \leq k$, induce the edge labels $\{20 i-10,1 \leq i \leq k\}=\{10,30, \cdots, 20 k-10\}$.

- The vertices $w_{i 1}$ and $w_{i}, 1 \leq i \leq k$, induce the edge labels $\{20 i-8,1 \leq i \leq k\}=\{12,32, \cdots, 20 k-8\}$.

- The vertices $v_{i}$ and $\left.v_{i 2}\right), 1 \leq i \leq k$, induce the edge labels $\{20 i-6,1 \leq i \leq k\}=\{14,34, \cdots, 20 k-6\}$.

- The vertices $w_{i 2}$ and $u_{i+1}, 1 \leq i \leq k$, induce the edge labels $\{20 i-4,1 \leq i \leq k\}=\{16,36, \cdots, 20 k-4\}$.

- The vertices $v_{i 2}$ and $u_{i+1}, 1 \leq i \leq k$, induce the edge labels $\{20 i-2,1 \leq i \leq k\}=\{18,38, \cdots, 20 k-2\}$.

- The vertices $w_{i}$ and $w_{i 2}, 1 \leq i \leq k$, induce the edge labels $\{20 i, 1 \leq i \leq k\}=\{20,40, \cdots, 20 k\}$.

So we obtain all the edge labels $\{2,4,6, \cdots, 20 k\}$.

Hence the subdivision of double triangular snakes $\left(2 \triangle_{k}\right.$-snake, $\left.k \geq 1\right)$ are even sequential harmonious.

Theorem 3.2. All subdivision of $2 m \triangle_{k}$-snake, $m, k \geq 1$ are even sequential harmonious.

Proof. Let $G=2 m \triangle_{k}$-snake has $q$ edges and $p$ vertices. The graph $G$ consists of the vertices $\left(u_{1}, u_{2}, \cdots, u_{k+1}\right),\left(v_{i}^{1}, v_{i}^{2}, \cdots, v_{i}^{m}\right)$, $\left(w_{i}^{1}, w_{i}^{2}, \cdots, w_{i}^{m}\right), 1 \leq i \leq k$. Therefore we get the generalised subdivision of double triangular snakes $S(G)$ by subdividing every edge of double triangular $2 m \triangle_{k}$-snake exactly once. Let $y_{i}$ be the newly added vertex between $u_{i}$ and $u_{i+1}$ while $w_{i 1}^{j}$ and $w_{i 2}^{j}$ are newly added vertices between $u_{i} w_{i}^{j}$ and $w_{i}^{j} u_{i+1}$ respectively where $1 \leq i \leq k$. Finally $v_{i 1}^{j}$ and $v_{i 2}^{j}$ are newly added vertices between $u_{i} v_{i}^{j}$ and $v_{i}^{j} u_{i+1}$ respectively, such that $1 \leq i \leq k, 1 \leq j \leq m$. Let the graph $S(G)$ as described as indicated in Figure 8.

We define the labeling function $f: V(S(G)) \rightarrow\{0,1,2, \cdots, 2 k(8 m+2)\}$ as follows: $f\left(u_{i}\right)=(4 m+2)(i-1), 1 \leq i \leq k+1=n$, $f\left(y_{i}\right)=(12 m+2) i-10 m-1,1 \leq i \leq k$, $f\left(v_{i}^{1}\right)=(4 m+2)(i-1)+4 l-2,1 \leq i \leq k, 1 \leq l \leq m$, $f\left(w_{i}^{j}\right)=(4 m+6)+(4 m+2)(i-1)+4(j-1), 1 \leq i \leq k, 1 \leq j \leq m$ 


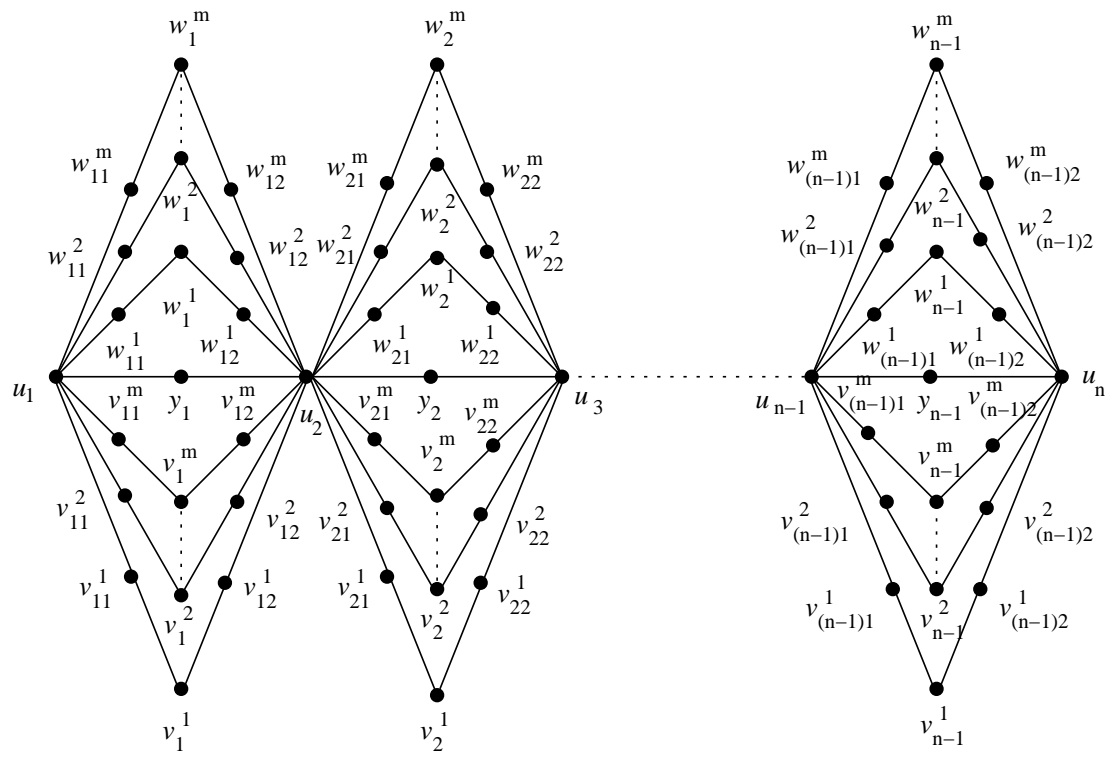

Figure 8: The subdivision of $2 m \triangle_{k^{-}}$snake

$f\left(w_{i j}^{j}\right)=(2 m-1)+(12 m+2)(i-1)+(4 m+2)(l-1)-2(j-1), 1 \leq i \leq k, 1 \leq$ $l \leq 2,1 \leq j \leq m$

$f\left(v_{i j}^{j}\right)=(4 m+1)+(12 m+2)(i-1)+(6 m+2)(l-1), 1 \leq i \leq k, 1 \leq l \leq 2$.

In a view of the above defined labeling pattern $f$ is even sequential harmonious for the graph $S(G)$. Hence $\mathrm{S}\left(2 m \triangle_{k}\right.$-snake) is even sequential harmonious for all $m \geq 1, k \geq 1$.

Illustration 3.3. An even sequential harmonious labeling of the graph subdivision of $6 \triangle_{3}$-snake is shown in Figure 9.

\section{References}

[1] A.Rosa.(1967) On certain Valuations of the vertices of graph,In theory of Graphs.International symposium,Rome,uly 1966,Gordon and breach.Newyork and Dunod,paris,Pp 349-355.

[2] R.L.Graham,N.J.A Sloane,(1980) On additive bases and harmonious graphs ,SIAM J. Algebr.disc.math., Vol 1,No 4,PP.382-404. 


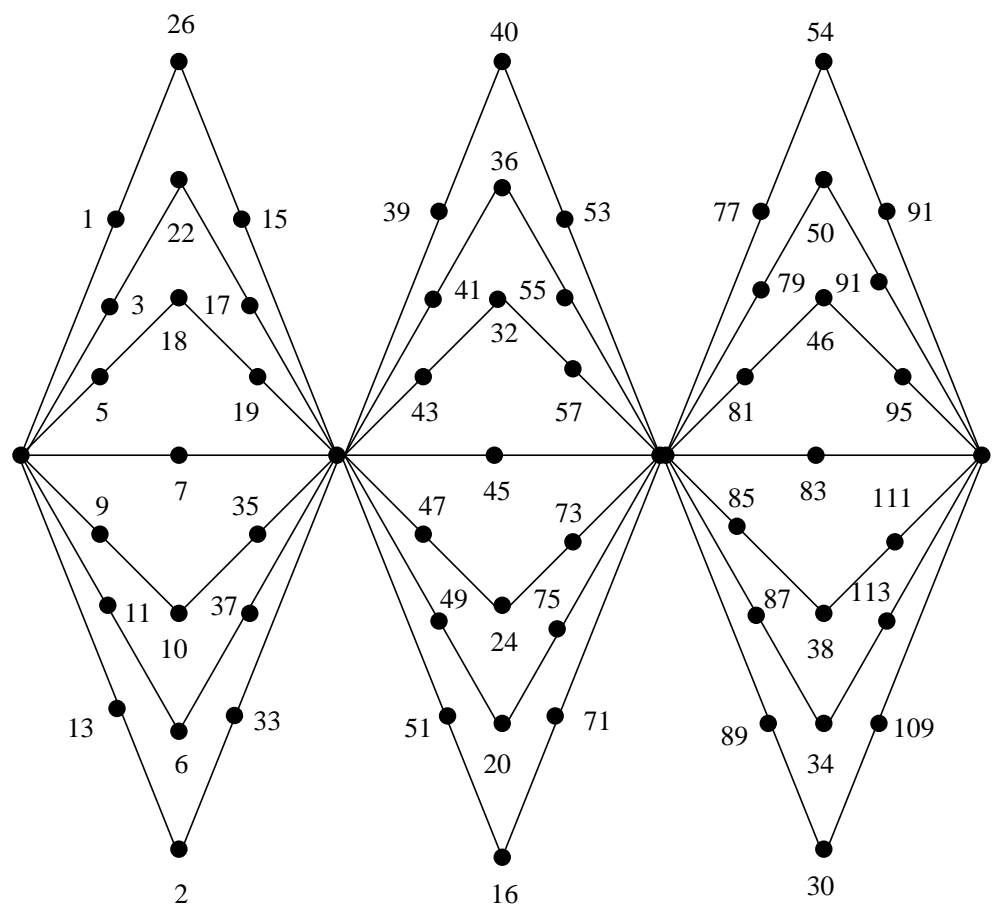

Figure 9: The graph subdivision of $6 \triangle_{3}$-snake with its even sequential harmonious labeling

[3] J.A.Gallian (2011) A Dynamic Survey of Graph Labeling, Electronic J.Combin.Fourteenth edition.

[4] F.Hararay (1969) Graph Theory, Addison Wesley,Reading MA.

[5] Z.Liang Z.Bai (2009) On the odd harmonious graphs with applications, J.Appl Math.Comput., 29,pp.105-116.

[6] Xi Yue,Yang Yuansheng and Wang Liping,(2008) On harmonious labeling of the double triangular n snake,IndianJ.pure apple Math., 39(2),pp. 177184.

[7] M.E.Abdel Adl Odd Harmonious labeling of cyclic snakes,Int. J. on application of graph Theoryin wireless ad hoc networks and sensor networks.

[8] A.Rosa,(1967) Cyclic Steiner Triple system and Labelings of Triangular Cacti, Scientia, 5, Pp.87-95. 
[9] D. Moulton,(1989) Graceful labelings of triangular snakes, Ars Combin. 28, Pp 3-13.

[10] S.D. Xu,(1995) Hatmonicity of triangular snakes, J. Math. Res. Exposition, 15, Pp 475-476. 
